\title{
Optimization and evaluation of motion sequences of an averaged human motion model by using kinematic techniques and new evaluation methods
}

\begin{abstract}
Signal processing, pattern recognition as well as modelling and simulation require a large amount of reference data, both for the development of new methods and for their evaluation. Depending on the application, the availability of databases is rather low. In the field of biosignal processing with a focus on the functionalization of furniture for nursing and hospital facilities, a database from a motion capturing system (MoCap), and a method to generate averaged human motion sequences was presented in subsequent works by our research group. Evaluations revealed that the averaged motion sequences partly contain artifacts caused by the averaging and thus are not directly usable. To use the averaged motion sequences e.g., in simulation tasks, this paper presents an extension with kinematic methods to combine averaged motion sequences and to suppress and thus optimize inappropriate motion artifacts by error correction. To check whether the results are usable after the processing steps, four evaluation criteria are proposed. The evaluation of the resulting motion sequences shows that sequences are generated which do not fully correspond to human motion sequences but are well suited for simulation tasks.
\end{abstract}

Keywords: average motion data, assessment of motion sequences, kinematics, motion capturing, evaluation criteria

https://doi.org/10.1515/cdbme-2021-2144

\footnotetext{
*Corresponding author: Andreas Kitzig: Niederrhein University of Applied Sciences, Faculty of Electrical Engineering and Computer Science, Ambient Intelligence Laboratory, Reinarzstr. 49, 47805 Krefeld, Germany, e-mail: andreas.kitzig@hsnr.de Julia Demmer, Edwin Naroska, Gudrun Stockmanns: Niederrhein University of Applied Sciences, Krefeld, Germany Reinhard Viga ${ }^{1}$, Anton Grabmaier ${ }^{1,2}$ : University of DuisburgEssen, Department of Electronic Components and Circuits ${ }^{1}$, Duisburg, Germany

Fraunhofer Institute for Microelectronic Circuits and Systems $(\mathrm{IMS})^{2}$, Duisburg, Germany
}

\section{Introduction}

MoCap systems have been continuously developed in recent years and their areas of application range from tasks in the field of animation [1] to applications in biosignal processing, e.g., gait analysis [2] or motion strategy analysis [3]. Our research focuses on biomechanical modelling in the field of functionalized nursing beds. A multi-stage modelling approach for the entire system "human + bed + sensor" has already been presented in [4]. Further work dealt with the creation of suitable motion sequences [5] for the use in simulation tasks and with motion data averaging, which enables general simulation independent of person-specific motion data [6]. The averaged motion data from [6] is based on a Hidden Markov Model (HMM) approach that provides models for single motions from which motion sequences can be combined. A disadvantage in [6] is the occurrence of additional unwanted artifacts in the resulting models. Furthermore, apart from a visual inspection, no evaluation of the motion sequence quality can be performed. To improve this, an approach to combine and optimize motion sequences using an error correction as well as new evaluation criteria are presented and discussed.

The discrete movement sequences resulting from the method for averaging human movements are briefly considered as basis, for further information refer to $[4,5,6]$. The resulting motion sequence $S=\left\langle\mathrm{p}_{1}, \mathrm{p}_{2}, \ldots, \mathrm{p}_{\mathrm{N}}\right\rangle$ of length $\mathrm{N}$ which is calculated from the HMM can be divided into individual discrete postures $\mathrm{p}_{\mathrm{i}}$ to describe a temporal sequence of movements. Each posture $p_{i}$ is represented by a stickman model ( 21 body points bp and angles $\varphi_{\mathrm{bp}}$ and $\theta_{\mathrm{bp}}$ as spherical coordinates/ spatial vectors $\overrightarrow{\mathrm{P}}_{\mathrm{bp}}$ ).

A comparison of the averaged movement sequences with real movement sequences from [5] shows that, besides the different durations of the movements, the averaged sequence has significantly less distinct angles and additional higher- 
frequency signal components that can be interpreted as a quick movement of the extremities. In addition, it may happen after averaging, that unnatural joint angles show up which cannot be assumed by a human being, for anatomical reasons. These effects can also occur in the context of recordings with a MoCap system (slipping of sensors, masking of markers in optical systems) and should be checked and corrected if necessary.

\section{Method}

First, we will consider how data sets can be checked and classified with respect to "normal" movements and postures. For this purpose, three criteria are defined to check the suitability of the motion sequences for a modelling application in general. The first criterion, "incomplete movements", can be detected by visual evaluation of the movement sequences and is characterized by movements ending abruptly in the course of time without a defined end position being reached.

The next criterion, "unexpected movements", describes movements that appear as tremors, twitches, or uncontrolled back-and-forth movements, especially of the extremities. In the temporal course, such effects are usually represented by sinusoidal signal components. The third criterion, "impossible movements", considers body postures that cannot be achieved from an anatomical point of view due to the maximum allowable joint angle of a human. These can be measured, for example, by analyzing the joint angles assumed in the posture.

\subsection{Combination and correction}

In the following, the approaches to optimize the disturbances described by the three criteria are presented. In this context, optimization is to be understood as the correction of errors that are contained in the sequences. Initially approaches of signal processing and kinematics in general have been considered, as they are used e.g., in the field of robotics for the optimization of the trajectory of a so-called end effector (e.g., tools on a robot arm). The approaches are combined to the method shown in Fig. 1 (1: combining sequences, 2: correcting the combined sequences) and motivated in the following by using the three criteria described above.

To complete missing end positions of a movement ("incomplete movements") in the averaged motion sequences and thus to optimize the sequence with respect to the kinematics and the process-oriented behaviour of humans, a procedure for interpolating the sequence between start and end position by means of cubic $\mathrm{C}^{2}$ splines is applied in parallel for

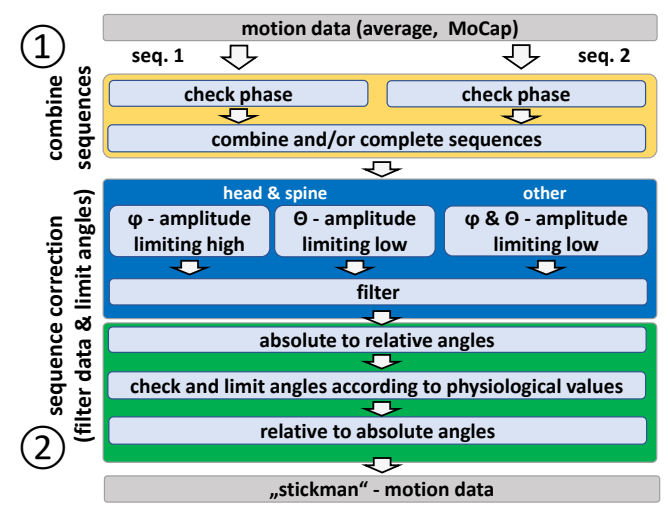

Fig. 1: Block diagram of the proposed method

all body parts of the stickman. This consideration is based on the assumptions that a healthy human performs a position change in a uniform sequence, which can be theoretically described as a goal-oriented coordinated process [8]. In addition, the method is used to combine two motion sequences into longer motion sequences from averaged motion data ("combine sequences" - 1, yellow/ Fig. 1).

The next point to consider is the occurrence of "unexpected movements". To compensate for this, a two-step procedure is proposed at this point, which modifies the data in the value and time domain. For this purpose, first, the temporal angular profiles for the angles $\varphi_{\mathrm{bp}}$ and $\theta_{\mathrm{bp}}$ for each element of the stickman are separated by a fast Fourier transformation (FFT) from the direct component (DC) and analyzed with respect to their individual dynamic range. Subsequently, attenuation of small amplitudes is performed using a given threshold value and then the addition of the DC to reproduce the original offset ("amplitude limiting low"-2, blue/ Fig. 1).

The angles of the head and spine are considered separately. Since averaging of these elements leads to sometimes large angular dynamics, large angles are reduced to an empirically found value of $10^{\circ}$. Furthermore, the dynamic range is analyzed and if the limited angles stand out as outliers, they are additionally attenuated ("amplitude limitation high" 2, blue/ Fig. 1). In this way, the interfering components can be minimized for each body part without significantly changing the structure of the useful components and the temporal structure of the motion sequence itself. In a final step, the angular sequences are smoothed with a low-pass filter to compensate for inconsistencies that may have arisen from the compression of small amplitudes ("filter" - 2, blue/ Fig. 1).

For compensation of "impossible movements", kinematic methods are used that consider the degrees of freedom and limitations of the joint structures involved. To compare the joints, joint positions and correlation angles with physiological considerations, the neutral-zero method is used [7]. Applying this to the stickman model, first the relative angles between the 
individual body parts of the stick figure are determined and then, if necessary, constrained by considering the minimum and maximum angles. (2, green/ Fig. 1)

\subsection{Evaluation criteria}

After presenting the method for combining and correcting motion sequences, evaluation criteria are presented below to be able to analyze the quality of the correction. After averaging, suitable ground truth data is no longer available, so a direct comparison with the input data is not possible. Therefore, we show how to compare the averaged motions with human motion patterns.

The evaluation of human movements is generally a challenge, as the evaluation is usually performed by trained experts using visual evaluation methods. This approach cannot easily be transferred to motion sequences from a database. To find objective criteria to assess movements it makes sense to consider automated processes and their procedures $[9,10]$.

A set of independent methods for the evaluation of motion is motivated by Fischer et al. [11]. Three of the criteria from [11] are adapted for this work. Furthermore, the body vector as an additional measure is presented. $\mathrm{x}, \mathrm{y}$, and $\mathrm{z}$ are Cartesian coordinates of the body. Fig. 2 shows examples of each evaluation method, these are considered in detail in the next section. The first criterion called "volume utilization VA(n)" is shown in (1). It describes the volume of the body $\left(\operatorname{area}(\mathrm{n})_{\mathrm{used},\{\mathrm{x}, \mathrm{y}, \mathrm{z}\}}\right)$ in relation to the environment $\left(\operatorname{area}(n)_{\max ,\{x, y, z\}}\right)$ and the change over time in samples $n$.

$$
\begin{gathered}
\operatorname{RA}(\mathrm{n})_{\{\mathrm{x}, \mathrm{y}, \mathrm{z}\}}=\left|\frac{1}{\operatorname{area}_{\max ,\{\mathrm{x}, \mathrm{y}, \mathrm{z}\}}-\operatorname{area}(\mathrm{n})_{\mathrm{used},\{\mathrm{x}, \mathrm{y}, \mathrm{z}\}}}\right| \\
\operatorname{VA}(\mathrm{n})=\operatorname{RA}(\mathrm{n})_{\mathrm{x}} \cdot \operatorname{RA}(\mathrm{n})_{\mathrm{y}} \cdot \operatorname{RA}(\mathrm{n})_{\mathrm{z}}
\end{gathered}
$$

Another criterion (2) is the body vector $\vec{P}_{K}$ which is oriented orthogonally to the stickman's torso (represented by
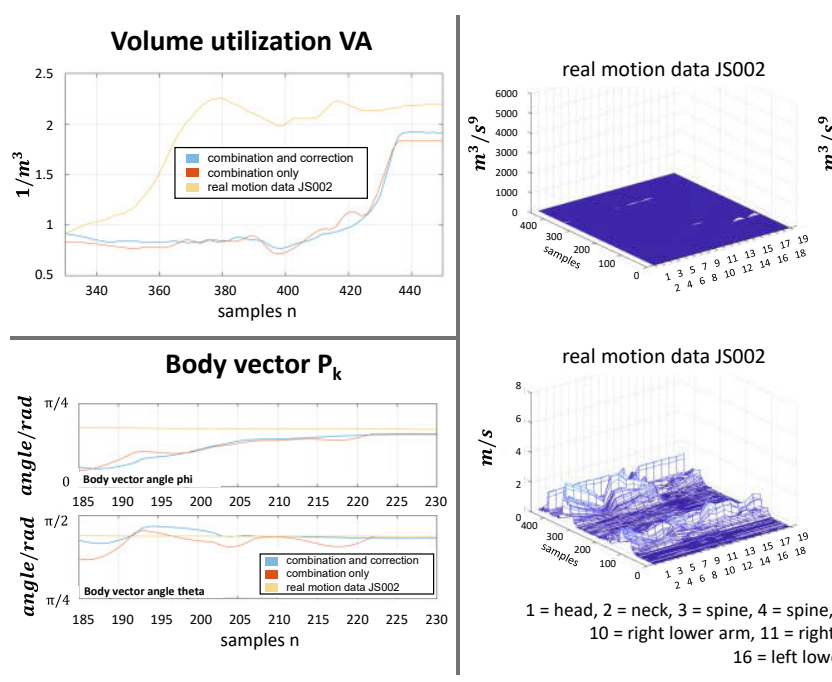

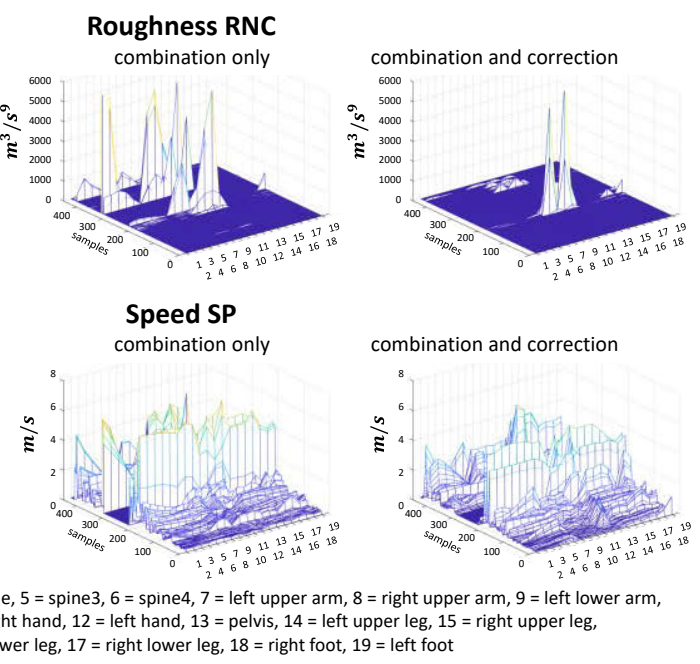

Speed SP
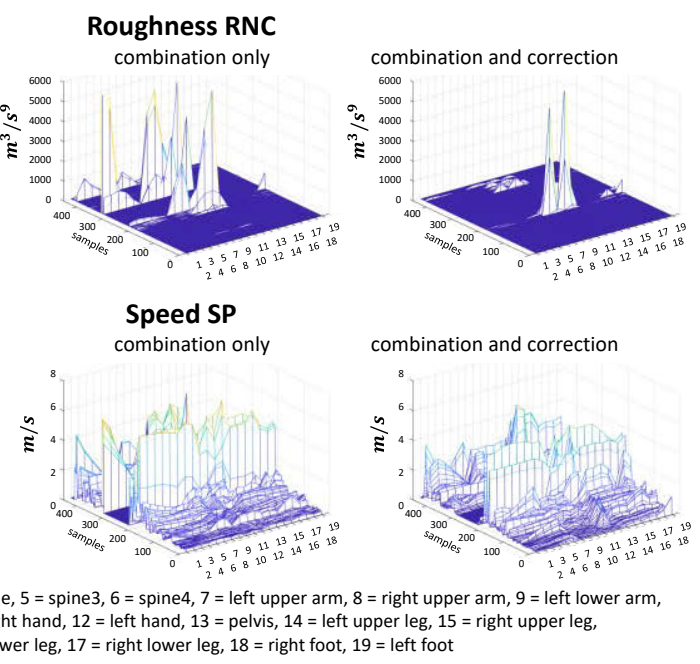

The next criterion (3) is called "roughness RNC(n)". This measure describes for each sample value n, how strongly the functional value in its environment changes per sample value. A high roughness is an indication that frequent and rapid changes of position in movement or location are taking place and is contrary to the required uniform movement of a human.

$$
\begin{gathered}
\operatorname{RN}(n)_{\{x, y, z\}}=\frac{d^{3}\{x(n-3: n), y(n-3: n), z(n-3: n)\}}{d^{3}} \\
\operatorname{RNC}(n)=\left|\operatorname{RN}(n)_{x}\right| \cdot\left|\operatorname{RN}(n)_{y}\right| \cdot\left|\operatorname{RN}(n)_{z}\right|
\end{gathered}
$$

The last criterion in (4) considers the instantaneous speed for a sample value $\mathrm{n}$ to regard the characteristic of a movement.

$\mathrm{SP}(\mathrm{n})=\frac{\sqrt{\sum\left[(\{(\mathrm{x}(\mathrm{n})-\mathrm{x}(\mathrm{n}-1)),(\mathrm{y}(\mathrm{n})-\mathrm{y}(\mathrm{n}-1)),(\mathrm{z}(\mathrm{n})-\mathrm{z}(\mathrm{n}-1))\})^{2}\right]}}{\mathrm{dt}}$

In comparison, high SP values can be assigned to a rather coarse movement sequence that has a lower quality.

\section{Evaluation and results}

Using an exemplary motion sequence ("supine - sit up - sitting - sit down - supine") from [5], the correct function of the method for combining and optimizing averaged motion sequences is evaluated and it is discussed how the new evaluation criteria can support the evaluation of motion sequences. The two analyzed averaged sequences were created from individual sequences using the method presented in Fig. 1, one as the original version (combination only - 1 in Fig.1) and one as the optimized version (combination and correction

Fig. 2: Volume utilization VA (left, upper), Body vector $P_{k}$-angles $\phi$ and $\theta$ (left, lower) and Roughness RNC and speed SP (right) 
- 1 and 2 in Fig. 1). For comparison, a corresponding real movement sequence (JS002 [5]) is used. The results for volume utilization in Fig. 2 (left, upper) shows that the basic sequence of all movements is comparable. However, the averaged sequences (blue and orange) have a higher number of additional movements. Fig. 2 (left, lower) shows a section of the temporal course of the body vector angles $\varphi$ and $\theta$. The results show that both artificially created sequences (blue and orange) differ from the body vector of the real data (yellow). The course can be described as much more "unsettled". It can also be seen that the averaged optimized course has less strong movement artefacts (blue). Furthermore, it becomes clear that the basic sequence is comparable apart from a different time structure for all three sequences. The results for SP and RNC are shown in Fig. 2 (right). No details are shown for space reasons, but it is clear from the overall view that all three structures differ. An optimum ratio of speed and roughness is shown in the real motion sequence (see Fig. 2 right, left). A low roughness coupled with a moderate velocity distribution can be seen, with the highest instantaneous velocities occurring in the extremities and head region. The two averaged sequences (Fig. 2 right, middle, and right) show considerably rougher courses in the evaluation and the movements of the individual body parts take place in comparison with higher speeds. Both criteria indicate that the movements are correspondingly more uneven and less smooth than real movements. It is also clear that the optimized movement has fewer speed steps and rough courses. These points were confirmed by a visual evaluation.

\section{Conclusion and outlook}

In the present work, a method for combining and optimising averaged motion sequences based on MoCap data was presented. Also, four criteria were proposed that can be used in addition to a visual evaluation of motion sequences. The presented method for combination and optimization was subjected to an evaluation against the four criteria and it has been shown that the resulting sequences are not fully comparable with real movements. However, it could also be shown that the combined sequences are close to real sequences. Although these are not equal, they can be used for simulation tasks because the artifacts they contain are relatively small compared to the non-optimized averaged sequences which are not usable for simulation. The presented evaluation criteria have proven to be suitable, were verified by visual comparisons and can contribute to a more objective evaluation of motion sequences. The optimization can be further improved, e.g., by analyzing the spectral composition of the angle courses to detect and suppress artefacts more specifically. Furthermore, it is useful to analyze the motion data in more detail [12].

\section{Author Statement}

The author state no funding involved. Authors state no conflict of interest. All persons included in this project have consented to informed consent.

\section{References}

[1] Sharma S, Verma S, Kumar M, Sharma L. Use of Motion Capture in 3D Animation: Motion Capture Systems, Challenges, and Recent Trends. COMITCon: 2019.

[2] Loose H, Orlowski K. Gait patterns in standard scenarios: Using Xsens MTw inertial measurement units. In: 6th International Conference on Research and Education in Mechatronics (REM), Bochum: 2015.

[3] Burget F, Maurer C, Burgard W, Bennewitz M. Learning motor control parameters for motion strategy analysis of Parkinson's disease patients. In: IEEE/RSJ International Conference on Intelligent RobotsSystems (IROS). Hamburg: 2015. pp. 5019-5025.

[4] Kitzig A, Stockmanns G, Viga R, Grabmaier A. Development of a multi-stage model for the determination of biosignal processing methods to derive vital signs from a force sensor functionalized nursing bed. In: BMT 2014;59

[5] Kitzig A, Schröter S, Naroska E, Stockmanns G, Viga R, Grabmaier A. MoveHN -- A database to support the development of motion based biosignal processing systems. In: 25th European Signal Processing Conference 2017.

[6] Kitzig A, Demmer J, Bolten T, Naroska E, Stockmanns G, Viga R, Grabmaier A. An HMM-based averaging approach for creating mean motion data from a full-body Motion Capture system to support the development of a biomechanical model. In: Cur. Dir. in Biom. Eng. 2018;4.

[7] Dittrich H, Schimmack M. Orthopädische Biomechanik, Springer-Verlag GmbH; 2019.

[8] Grosser M, Hermann H, Tusker F. Die sportliche Bewegung. Blv Buchverlag, 1987.

[9] Parisi Gl, Magg S, Wermter S. Human motion assessment in real time using recurrent self-organization. In: 25th IEEE International Symposium on Robot and Human Interactive Communication (RO-MAN), 2016.

[10] Endres F, Hess J, Burgard W. Graph-Based Action Models for Human Motion Classification. In ROBOTIK 2012; 7th German Conference on Robotics, 2012.

[11] Fischer CA, Kondraske GV. A new approach to human motion quality measurement. In: Proceedings of the 19th Annual International Conference of the IEEE Engineering in Medicine and Biology Society. 'Magnificent Milestones and Emerging Opportunities in Medical Engineering' (Cat. No.97CH36136), 1997.

[12] Demmer J, Kitzig A, Stockmanns G, Naroska E, Viga R, Grabmaier A. Adaptation of cluster analysis methods to optimize a biomechanical motion model of humans in a nursing bed. In: 28th European Signal Processing Conference (EUSIPCO). Amsterdam, 2020 